\title{
Different Therapeutic Paths (Colchicine vs. Anakinra) in Two Patients With Schnitzler's Syndrome
}

\author{
Marko BAREŠIĆ, ${ }^{1}$ Joško MITROVIĆ, ${ }^{2}$ Jadranka MOROVIĆ VERGLES, ${ }^{2}$ Branimir ANIĆ ${ }^{1}$ \\ ${ }^{1}$ Department of Internal Medicine, Division of Clinical Immunology and Rheumatology, \\ University Hospital Center Zagreb, Zagreb, Croatia \\ ${ }^{2}$ Department of Internal Medicine, Division of Clinical Immunology and Rheumatology, \\ Dubrava University Hospital, Zagreb, Croatia
}

\begin{abstract}
Schnitzler's syndrome is a rare autoinflammatory syndrome with unidentified mechanism of disease and etiology with unknown definitive treatment algorithm. The two obligatory criteria for the diagnosis of Schnitzler's syndrome include chronic urticarial rash and monoclonal gammopathy (immunoglobulin $\mathrm{M}$ or immunoglobulin $\mathrm{G}$ ). In this article, we describe two patients with different courses of disease with different average lengths of time between initial symptoms and the final diagnosis ( 6 months to 8 years). Exclusion of more common conditions is needed to ensure the correct diagnosis. Treatment strategy depends on the patient's constitutional symptoms (fever, malaise, generalized myalgia, and arthralgias) and laboratory tests of inflammation. Treatment includes usage of conventional drugs and cytokine blockade (interleukin-1 and interleukin-6). Further studies are needed to determine the precise mechanism of disease and the appropriate targeted therapy.

Keywords: Anakinra; canakinumab; colchicine; glucocorticoids; Schnitzler's syndrome.
\end{abstract}

Schnitzler's syndrome is an uncommon disorder characterized by urticarial rash and monoclonal gammopathy. It was first described by a French dermatologist Lilian Schnitzler and named after her. ${ }^{1}$ The disease is thought to be an adult onset autoinflammatory syndrome with unknown etiology and unknown pathogenesis. ${ }^{2,3}$ The diagnosis is reached after excluding all other more common causes ${ }^{4}$ taking into account the Strasbourg diagnostic criteria (Table 1). ${ }^{2}$ Treatment strategies depend on several factors, the most important being the general condition of the patient and levels of acute phase reactants. In this article, we aimed to evaluate the different treatment strategies by comparing the clinical courses of two patients, one achieving remission with colchicine and the other with interleukin 1 (IL-1) receptor blockade after failing to respond to the conventional immunosuppressants.

\section{CASE REPORT}

Case 1- A 50-year-old female patient with unremarkable medical history had an eight-year history of intermittent urticarial rash (localized, later on generalized) and periodic high fevers with chills and shivering, accompanied by generalized myalgia and malaise. Laboratory work-up registered elevated acute phase reactants, leukocytosis with neutrophilia, and elevated immunoglobulin M (IgM). Tests for antinuclear antibodies, complement levels, C1 esterase inhibitor, anti-neutrophil cytoplasmic antibodies, $\mathrm{IgE}$ and eosinophil cationic protein were negative. Skin allergy and inhaled allergens testing were also unremarkable. We treated the patient with antihistamines with no effect. During a routine check-up, we detected IgM kappa monoclonal gammopathy and performed additional tests 
Table 1. Strasbourg diagnostic criteria for Schnitzler's syndrome

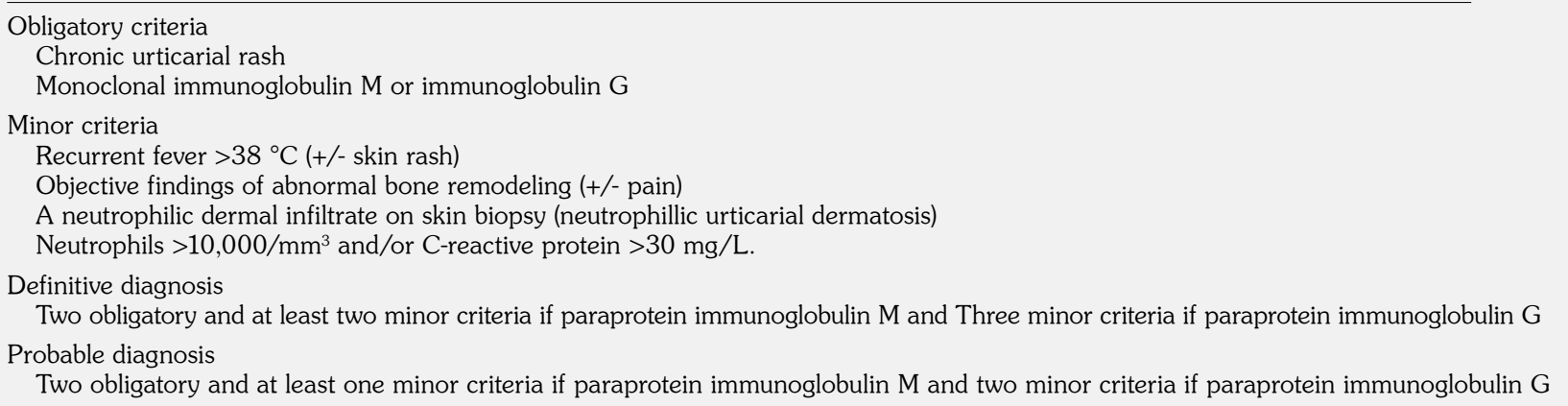

(analysis of bone marrow, computed tomography of the thorax, abdomen, and pelvis) and excluded lymphoproliferative disease. We controlled the urticarial rash and high fever only with higher doses of prednisone ( $0.5 \mathrm{mg}$ per $\mathrm{kg}$ or more). The attempt to use other immunosuppressants (azathioprine, cyclophosphamide, mycophenolate mofetil) as steroid sparing drugs was also ineffective. The patient developed side effects of glucocorticoid therapy - diabetes mellitus and depression - and stopped the regular follow-up visits and had to retire. Two years later, she was hospitalized during a period of prolonged high fever and generalized urticarial rash. Once again, we performed detailed work-up. Skin biopsy showed elements of neutrophilic dermatosis (Figure 1). After excluding all other possible causes of the symptoms, we reached a diagnosis of Schnitzler's syndrome. Due to prominent general constitutional symptoms and ineffectiveness of antihistamines, several immunosupressants and steroid-dependent disease the treatment with anakinra was started with a prompt and excellent result. Patient has been in remission in the followup period with no need for prednisone.

Case 2- A 53-year-old male patient with unremarkable medical history had a four-month history of acute generalized rash followed by high fever, shivers, and hives. Higher doses of parenteral or oral glucocorticoids (0.3-0.5 mg per $\mathrm{kg}$ ) reduced the symptoms. Due to elevated acute phase reactants (erythrocyte sedimentation rate and C-reactive protein), we performed check-up and excluded infection. Standard nutritive and allergen inhalation tests were negative. We treated the patient with prednisone and azathioprine as a steroid sparing drug with little effect. Further workup in the rheumatology unit was also negative for antinuclear antibodies, systemic antineutrophil

(a)
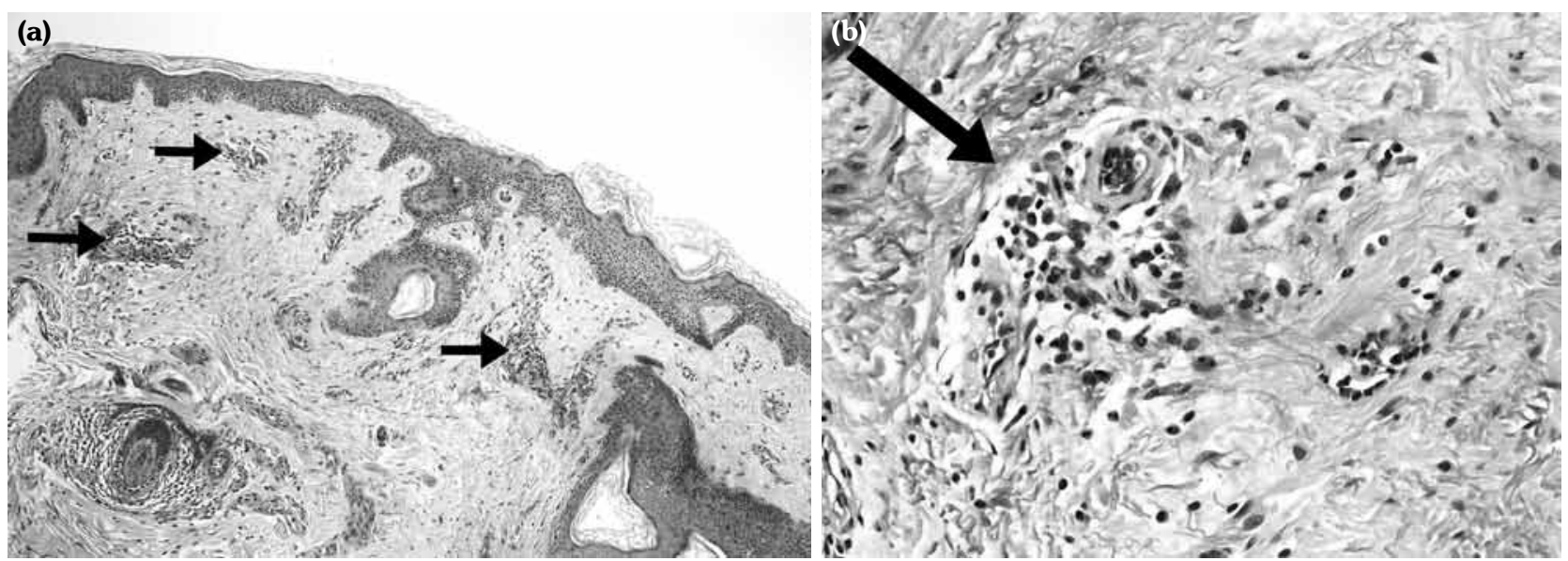

Figure 1. Skin biopsy - Periodic acid-Schiff staining - perivascular and intravascular cell infiltrates, predominantly neutrophils (arrows). (a) 100x magnification, (b) 400x magnification. 
cytoplasmic antibody-associated vasculitides and there was no decrease in complement levels. After obtaining the finding of IgM kappa monoclonal gammopathy, additional tests (bone marrow analysis and computed tomography) ruled out lymphoproliferative disease. Having reviewed all the symptoms, we reached a diagnosis of Schnitzler's syndrome. Due to relatively mild general constitutional symptoms, we started the patient on colchicine and sustained a low dose of prednisone with rapid improvement of rash. The patient has been tolerating colchicine well and has remained stable with almost normal acute phase reactants in the follow-up period.

\section{DISCUSSION}

Schnitzler's syndrome, being a rare disease, is often overlooked and not frequently included in the differential diagnosis of patients presenting with urticarial rash.

According to the literature, the time elapsed from onset of symptoms to diagnosis is approximately five years. ${ }^{2,3}$ The first patient we described had a latency of more than eight years before the correct diagnosis was established.

Both described patients had similar presenting symptoms (periodic urticarial rash and fever) but with different symptom intensity and course of disease. They presented with both major criteria (chronic urticarial rash and monoclonal gammopathy) and the majority of minor criteria needed for the diagnosis of Schnitzler's syndrome..$^{2,3}$

Rash (red macules, papules or plaques without itching, with burning sensation) is present in almost all patients with Schnitzler's syndrome and can occur on any part of the body. ${ }^{5}$ Monoclonal IgM component is the second most important criteria; it is associated with kappa light chains in $90 \%$ of patients. Differential diagnostic procedure should be carried out to exclude lymphoproliferative disease such as multiple myeloma and Waldenstrom's macroglobulinemia. ${ }^{6}$ Both described patients underwent detailed check-up to exclude the hematologic disease.

In the diagnostic procedure, skin biopsy plays an important role especially in excluding the diagnosis of urticarial vasculitis with the predominant elements of leukocytoclastic vasculitis. ${ }^{3,5}$

General constitutional symptoms and their effect on patient's quality of life determine the treatment strategy - anakinra for patients with more severe clinical and laboratory signs and colchicine for less prominent clinical and laboratory signs of the disease. Although the mechanism of the disease is still unknown, the efficacy of IL-1 blockade suggests that Schnizler's syndrome is an IL-1 driven disorder. ${ }^{7,8}$ Long-term efficacy and safety of anakinra have been proven in a cohort of patients. ${ }^{9}$

Positive response to colchicine in our patient with less pronounced systemic constitutional symptoms could suggest colchicine as a drug of first choice in the treatment of Schnitzler's syndrome. In case of primary or secondary inefficacy of colchicin, blockade of IL-1 receptor (anakinra) and IL-1 beta receptor (canakinumab) should be tried. ${ }^{10}$ Inhibition of IL- 6 receptor with tocilizumab was proved to be useful in certain patients supporting the role of IL-6 in the pathogenesis of the disease. ${ }^{11}$ Positive effects of colchicine treatment suggest yet unknown mechanisms of the action of colchicine, prompting further studies to clarify the role of the drug in the therapy of Schnitzler's syndrome.

Regular follow-ups of patients with Schnitzler's syndrome are warranted due to higher risk for the development of secondary amyloidosis and lymphoproliferative disorders especially in untreated and undertreated patients. ${ }^{12}$

In conclusion, this article reports two diverse clinical courses and different managements of patients with Schnitzler's syndrome, and indicates that treatment strategies dependent on the patient's general condition and levels of acute phase reactants. According to our limited experience, colchicine may be used as the drug of first choice in the treatment of Schnitzler's syndrome and IL-1 blockade may be used as the second line therapy in refractory Schnitzler's syndrome patients in whom other immunosuppressants fail.

\section{Declaration of conflicting interests}

The authors declared no conflicts of interest with respect to the authorship and/or publication of this article. 


\section{Funding}

The authors received no financial support for the research and/or authorship of this article.

\section{REFERENCES}

1. Schnitzler L: Lésions urticariennes chroniques permanentes (érythème pétaloïde?). J Dermatol Angers; 1972.

2. Simon A, Asli B, Braun-Falco M, De Koning $\mathrm{H}$, Fermand JP, Grattan C, et al. Schnitzler's syndrome: diagnosis, treatment, and follow-up. Allergy 2013;68:562-8.

3. Lipsker D. The Schnitzler syndrome. Orphanet J Rare Dis 2010;5:38.

4. Markotic A, Skerk V, Cvetko Krajinovic L, Beus A. Is the clinical picture of Schnitzler syndrome always Schnitzler syndrome? Clin Exp Rheumatol 2009;27:507-9.

5. Sokumbi O, Drage LA, Peters MS. Clinical and histopathologic review of Schnitzler syndrome: the Mayo Clinic experience (1972-2011). J Am Acad Dermatol 2012;67:1289-95.

6. Tinazzi E, Puccetti A, Patuzzo G, Sorleto M, Barbieri A, Lunardi C. Schnitzler syndrome, an autoimmune- autoinflammatory syndrome: report of two new cases and review of the literature. Autoimmun Rev 2011;10:404-9.

7. Koch A, Tchernev G, Chokoeva AA, Lotti T, Wollina $\mathrm{U}$. Schnitzler syndrome responding to interleukin-1 antagonist anakinra. J Biol Regul Homeost Agents 2015;29(1 Suppl):19-21.

8. Besada E, Nossent H. Dramatic response to IL1RA treatment in longstanding multidrug resistant Schnitzler's syndrome: a case report and literature review. Clin Rheumatol 2010;29:567-71.

9. Néel A, Henry B, Barbarot S, Masseau A, Perrin F, Bernier $\mathrm{C}$, et al. Long-term effectiveness and safety of interleukin-1 receptor antagonist (anakinra) in Schnitzler's syndrome: a French multicenter study. Autoimmun Rev 2014;13:1035-41.

10. de Koning HD, Schalkwijk J, Stoffels M, Jongekrijg J, Jacobs JF, Verwiel E, et al. The role of interleukin-1 beta in the pathophysiology of Schnitzler's syndrome. Arthritis Res Ther 2015;17:187.

11. Krause K, Feist E, Fiene M, Kallinich T, Maurer M. Complete remission in 3 of 3 anti-IL-6-treated patients with Schnitzler syndrome. J Allergy Clin Immunol 2012;129:848-50.

12. de Koning HD. Schnitzler's syndrome: lessons from 281 cases. Clin Transl Allergy 2014;4:41. 